\title{
On the uncertainty in the nuclear model calculation of neutron and proton induced reaction cross sections
}

\author{
L. Mercatalia , A.Yu. Konobeyev, and C.H.M. Broeders \\ Institute for Reactor Safety, Forschungszentrum Karlsruhe, GmbH 76021, Karlsruhe, Germany
}

\begin{abstract}
The paper deals with the investigation of the uncertainty associated to the calculation of activation and transmutation cross sections for neutron and proton induced reactions. Results of the calculations performed by means of the TALYS code and the ALICE/ASH code with different models for the description of the nuclear level densities have been extensively compared with EXFOR experimental data. Statistical deviation factors quantifying the discrepancy between experimental and theoretical results have been obtained, which can be used to define appropriate models for the nuclear reaction cross section calculation for different mass range of target nuclei. Furthermore, experimental data have been also compared against the most modern nuclear data files in order to investigate the gain in accuracy one should expect from the evaluation work with respect to the data obtained via nuclear model calculation.
\end{abstract}

\section{Introduction}

Nuclear model calculations play nowadays a relevant role in the generation of nuclear data files in particular in the high energy region $(\mathrm{E}>1 \mathrm{MeV})$ where the large amount of open channels imposes an extensive use of theoretical predictions. As a consequence, outstanding improvements in the field of cross section theory have been achieved within the last decades. However, despite such big developments a methodology to accurately calculate every nuclear cross section for any target nuclei over a wide energy range is not yet available. A systematic assessment of the predictive capabilities of the most advanced nuclear models and computational tools having a direct relation in the generation of nuclear data files is still needed. In this paper such an assessment is presented with regard to the use of different models for the representation of the nuclear level densities at the equilibrium states, these being both phenomenological and microscopic ones. Results of the theoretical predictions have been compared against experimental data and the discrepancy is quantified by means of statistical deviation factors, which can be used to provide recommendations to the users on the best combinations of codes and models to optimize the accuracy of the simulations.

\section{Experimental data}

All the neutron and proton experimental data to be compared with the theoretical predictions have been extracted from EXFOR and presented in the $\mathrm{C} 4$ format by means of a processing procedure performed using the X4TOC4 code [1]. As a general criterion, obsolete and superceded measurements, measurements for targets containing natural mixture of isotopes as well as data for reactions with metastable products were excluded from the consideration. The preliminary comparison with calculations has shown a number of errors in the

a Presenting author, e-mail: mercatali@irs.fzk.de compilation of the EXFOR files, which have been reported to the NDS, IAEA. Data have been subsequently corrected or deleted form the consideration.

\subsection{Neutron induced reactions}

All the (n,xnypz $\alpha$ ) reactions available in EXFOR have been analyzed with the exception of $(n, \gamma),(n, n p),(n, d)$ and $\left(n,{ }^{3} \mathrm{He}\right)$. A total of 17937 experimental points have been considered, for target nuclei with atomic number from 13 to 83 . The energy of the projectile considered is in the range $0.1 \mathrm{MeV} \div$ 64.4 MeV. As far as the energy distribution of the experimental points (fig. 1), $30 \%$ of the total measurements considered are relative to the projectile energy in the range $14 \div 15 \mathrm{MeV}$.
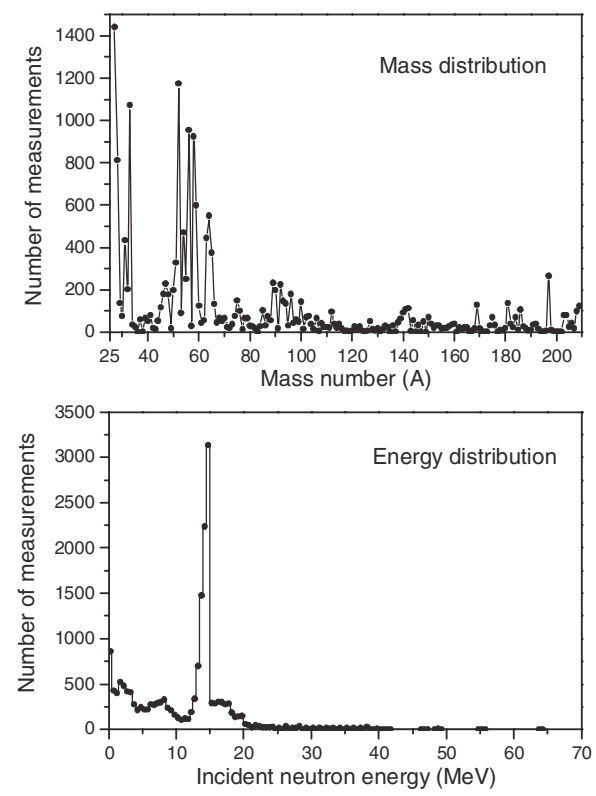

Fig. 1. Distributions of experimental points extracted from EXFOR for neutron induced reactions. 

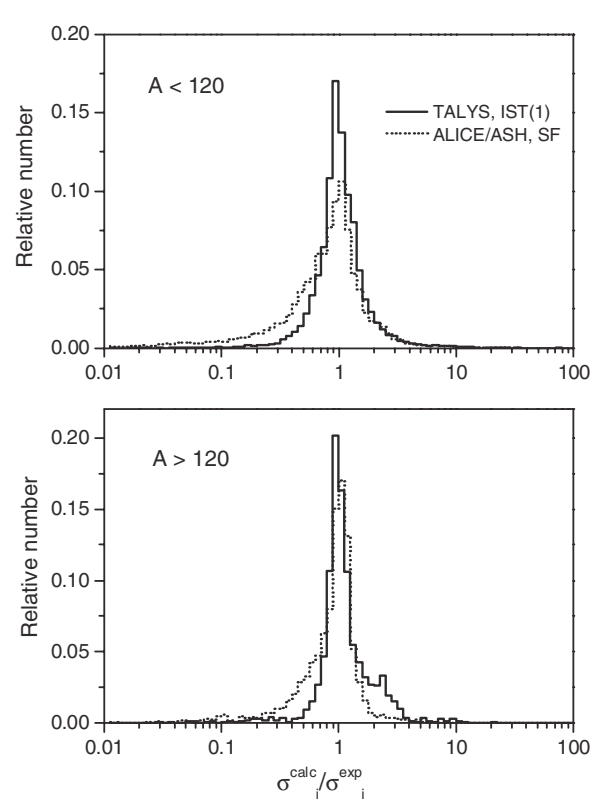

Fig. 2. Ratio of calculated cross sections to measured cross sections obtained using the IST-1 and SF models.

\subsection{Proton induced reactions}

Measured independent non-cumulative yields of radionuclides for $(\mathrm{p}, \mathrm{x})$ reactions including the radioactive capture of target nuclei with $12 \leq \mathrm{Z} \leq 83$ and $24 \leq \mathrm{A} \leq 209$ at proton energies from the minimal available up to $150 \mathrm{MeV}$ were selected for the comparison. The following data have been excluded from the consideration: i) sums of cross sections for various reactions, ii) data averaged for a wide range of the proton incident energy, and iii) measurements for thick targets. As a result, 735 proton induced reactions $(\mathrm{p}, \mathrm{x})$ have been investigated, relative to 162 target nuclei. The total number of experimental points is equal to 19691 and about $50 \%$ of these are relative to the energy of the projectile lower than $50 \mathrm{MeV}$.

\section{Theoretical predictions}

All the cross section calculation have been performed by means of the TALYS [2] code and the ALICE/ASH [3] code, which are being used quite extensively within the international community for the generation of nuclear data files.

As far as the TALYS code, the pre-equilibrium particle emission is described using the two component exciton model [4]. The model implements new expressions for internal transition rates and new parameterization of the average square matrix element for the residual interaction obtained using the optical model potential of Koning et al. [5]. The phenomenological model is used for the description of the preequilibrium complex particle emission [6]. The contribution of direct processes in inelastic scattering is calculated using the ECIS-97 code included in TALYS as a subroutine. The equilibrium particle emission is described by means of the Hauser-Feshbach model.

The geometry dependent hybrid model (GDH) [7] and the Weisskopf-Ewing model were applied for numerical calculations with the ALICE/ASH code, which is a modified and advanced version of the ALICE code [8]. Intranuclear transition rates were calculated using the effective cross section of nucleon-nucleon interactions in nuclear matter. Corrections were made to the GDH approach for the treatment of effects in peripheral nuclear regions $[9,10]$. The multiple precompound emission is described by the approximate approach [7]. The exciton state density is calculated taking into account pairing corrections, the correction for the Pauli principle and the final depth of the nuclear potential well for the exciton state $n=3$. The number of neutrons and protons for initial exciton state was calculated using realistic nucleon-nucleon interaction cross sections in nucleus [9]. The exciton coalescence pick-up model $[11,12]$ and the knock-out model [13] were used for the description of the pre-equilibrium complex particle emission.

The goal of this work is to investigate the impact of the different models for the description of the nuclear level densities on the results of the cross section calculation. For the purposes of the present work, both the TALYS and the ALICE/ASH codes have been used with default values of input parameters with the exception of the parameters describing the particular model used for the nuclear level densities description. In particular, six different level density models have been considered, corresponding to the input parameters ldmodel equal to 1,2 or 3 and ldopt equal to 0,4 and 5 in the TALYS code and in the ALICE/ASH code respectively. Detailed information on these models can be found in [14-16]. The main features can be summarized as follows:

- ldmodel1: Fermi gas model with the energy dependent level density parameter $a(U)$ without explicit description of the collective enhancement,

- ldmodel2: Fermi gas model with the energy dependent level density parameter $a(U)$ with explicit description of the rotational and vibrational enhancement,

- ldmodel3: Microscopic model based on the results of microscopic calculations performed by Goriely et al. using the Hartree-Fock-BCS model,

- ldopt0: Fermi gas model with the dependent level density parameter $a=A / 9$,

- ldopt4: Fermi gas model with the energy dependent level density parameter $a(U)$,

- ldopt5: Superfluid nuclear model.

We will refer to the results of the calculations performed with the different models using the following notation: ldmodel $1=$ IST1, ldmodel $2=$ IST $-\mathrm{C}$, ldmodel $3=\mathrm{G}$, ldopt $0=\mathrm{FG}$, ldopt $4=$ IST2, ldopt $5=\mathrm{SF}$. In order to avoid inconsistencies between the results of the TALYS and the ALICE/ASH calculations caused by different values of total non-elastic cross sections, the ALICE/ASH calculated cross sections have been normalized to the values calculated by the TALYS code.

The discrepancies in between theoretical predictions and experimental data are quantified by means of different deviation factors, as follows:

$$
H=\left(\frac{1}{N} \sum_{i=1}^{N}\left(\frac{\sigma_{i}^{\mathrm{exp}}-\sigma_{i}^{\text {calc }}}{\Delta \sigma_{i}^{\exp }}\right)^{2}\right)^{1 / 2}
$$


Table 1. Deviation factors for neutron induced reactions.

\begin{tabular}{ccccccc}
\hline Factors & IST(1) & IST-C & G & FG & IST(2) & SF \\
\hline H & 10.3 & 30.6 & 12.6 & 16.2 & 28.9 & 13.8 \\
R & 1.3 & 1.6 & 1.3 & 1.05 & 0.8 & 1.0 \\
D & 0.50 & 1.0 & 0.6 & 0.5 & 0.6 & 0.5 \\
F & 2.09 & 2.9 & 2.1 & 2.8 & 18.3 & 3.5 \\
L & 0.14 & 0.6 & 0.2 & 0.3 & 0.6 & 0.2 \\
\hline
\end{tabular}

Table 2. Deviation factors for proton induced reactions.

\begin{tabular}{ccccccc}
\hline Factors & IST(1) & IST-C & G & FG & IST(2) & SF \\
\hline H & 24.4 & 103.0 & 25.3 & 778.4 & 1006 & 57 \\
R & 1.7 & 2.0 & 1.8 & 4.4 & 12.0 & 3.7 \\
D & 1.0 & 1.3 & 1.05 & 3.8 & 11.5 & 3.1 \\
F & 2.1 & 2.6 & 2.2 & 3.5 & 12.7 & 4.7 \\
L & 0.9 & 0.9 & 0.9 & 1.0 & 0.9 & 0.9 \\
\hline
\end{tabular}

$$
\begin{gathered}
R=\frac{1}{N} \sum_{i=1}^{N} \frac{\sigma_{i}^{\text {calc }}}{\sigma_{i}^{\exp }} \\
D=\frac{1}{N} \sum_{i=1}^{N}\left|\frac{\sigma_{i}^{\exp }-\sigma_{i}^{c a l c}}{\sigma_{i}^{\exp }}\right| \\
F=10^{\left(\frac{1}{N} \sum_{i=1}^{N}\left[\log \left(\sigma_{i}^{\exp }\right)-\log \left(\sigma_{i}^{\text {calc }}\right)\right]^{2}\right)^{1 / 2}}
\end{gathered}
$$

where $\sigma_{i}^{\text {exp }}$ and $\Delta \sigma_{i}^{\text {exp }}$ are the measured cross section and its uncertainty, $\sigma_{i}^{\text {calc }}$ is the calculated cross section, $\mathrm{N}$ is the number of experimental points.

To estimate the uncertainty in the calculated cross sections a covariance matrix has been proposed, which takes into account the contribution due to the failure of the model used for the calculations [17]. The matrix, which defines the "model deficiencies", is constructed using the mean model error $\delta u$ extracted from the reproduction of experimental data by a given reaction model as follows:

$$
M_{i, j}^{(d e f)}=C_{i, j}(\delta u)^{2} \sigma_{i}^{\text {calc }}\left(E_{i}\right) \sigma_{j}^{\text {calc }}\left(E_{j}\right)
$$

where $E_{i}$ and $E_{j}$ are kinetic energies of primary particles and coefficients $C_{i, j}$ are defined in [16]. The square of the mean model error is used in the present work as an additional factor to estimate the quality of model calculations, as follows:

$$
L=\left[\sum_{i=1}^{N}\left(\frac{\sigma_{i}^{\text {calc }}}{\Delta \sigma_{i}^{\exp }}\right)^{2}\left(\frac{\sigma_{i}^{c a l c}-\sigma_{i}^{\exp }}{\sigma_{i}^{c a l c}}\right)^{2} / \sum_{i=1}^{N}\left(\frac{\sigma_{i}^{\text {calc }}}{\Delta \sigma_{i}^{\exp }}\right)^{2}\right] .
$$

\section{Results}

The limited volume of the paper allows the presentation of only a part of the results. Details information can be additionally found in $[18,19]$.

Tables 1 through 3 provide a summary of the main findings of our work which can be used as preliminary recommendations on the use of the various nuclear models implemented
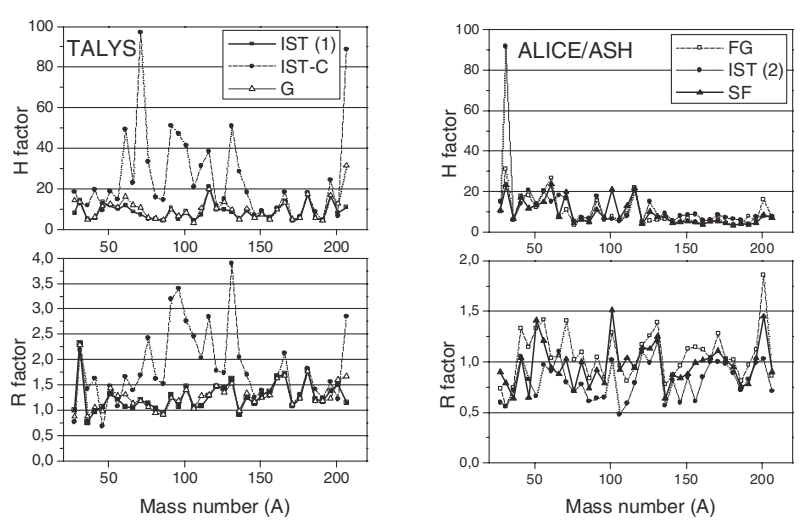

Fig. 3. The $H$ and $R$ factors calculated with TALYS and ALICE/ASH in the case of neutron induced reactions.

in the TALYS and ALICE/ASH codes. All the TALYS calculations have been performed with the code version 0.64. However, preliminary investigations concerning the use of the recently released updated version 0.72 did not show relevant changes on the calculated factors.

When averaging the factors of equations (1-4) and equation (6) calculated for all the target nuclei over the entire energy range and over all the channels, the minimal values of the factors were obtained using the IST1 model (tables 1 and 2). In addition, table 3 provides also results relative to two different mass number ranges of the target nuclei. Approximately, the division corresponds to the dominate contribution of equilibrium $(\mathrm{A}<120)$ and precompound $(\mathrm{A}>120)$ processes in the $(n, p)$ and $(n, \alpha)$ reactions, which together represent nearly $58 \%$ of the total experimental points considered in this work. For neutron induced reactions it was found that the accuracy in the prediction with the TALYS code is higher with respect to the one achieved using ALICE/ASH in the range of target nuclei with $A<120$. In particular, the IST1 model and the $G$ model applied for the nuclear level density calculation shows the best results in the case of neutron induced reactions and proton induced reactions respectively. On the contrary, the minimal $\mathrm{H}$ value in the target mass number range $\mathrm{A}>120$ corresponds to the use of the ALICE/ASH code with the superfluid model (SF). In figure 3 the $\mathrm{H}$ and $\mathrm{R}$ deviation factors are plotted as functions of the target nuclei mass number. As far as the TALYS calculations, we found that the IST1 and the G models are somehow in competition, while the IST-C model appears to be the less accurate. In the case of the ALICE/ASH calculations, SF is the model with the best capability for prediction of the experimental data. The results relative to the analysis of the proton induced reactions have been used to generate the PADF library [20,21].

\subsection{Comparison of theoretical predictions with evaluated data libraries}

A comparison between experimental data and evaluated data has also been performed in order to quantify the gain in accuracy due to the evaluation process with respect to the uncertainty due to the nuclear model calculations. Measured cross sections have been compared with the most complete 
Table 3. Deviation factors for neutron $\left(\mathrm{H}_{n}\right)$ and proton $\left(\mathrm{H}_{p}\right)$ induced reactions.

\begin{tabular}{ccccccc}
\hline Factors & IST(1) & IST-C & $\mathrm{G}$ & $\mathrm{FG}$ & IST(2) & $\mathrm{SF}$ \\
\hline \multicolumn{7}{c}{ Target nuclei with $\mathrm{A}<120$} \\
$\mathrm{H}_{n}$ & 10.3 & 29.3 & 12.0 & 17.5 & 31.4 & 14.9 \\
$\mathrm{H}_{p}$ & 22.0 & 111.6 & 20.7 & 851.6 & 1100.6 & 625 \\
$\mathrm{H}_{n}$ & 10.4 & 36.4 & 15.3 & 6.1 & 7.4 & 5.4 \\
$\mathrm{H}_{p}$ & 34.2 & 34.5 & 41.5 & 35.0 & 23.5 & 16.2 \\
\hline
\end{tabular}

Table 4. Deviation factors for neutron induced reactions calculated using evaluated cross sections from different nuclear data libraries.

\begin{tabular}{ccccc}
\hline Factors & ENDF/B-VI.8 & $\begin{array}{c}\text { ENDF/B-VII } \\
\text { (beta 1) }\end{array}$ & JEFF-3.0/A & JENDL-3.3 \\
\hline $\mathrm{H}$ & 9.2 & 9.5 & 6.9 & 8.2 \\
$\mathrm{R}$ & 1.1 & 1.7 & 1.1 & 1.6 \\
$\mathrm{D}$ & 0.3 & 0.8 & 0.4 & 0.8 \\
\hline
\end{tabular}

and modern evaluated data sets. Results of such comparison are summarized in table 3. Data from JEFF-3.0/A (European Activation File [22]) present minimal values of deviation factors when compared with other libraries.

\section{Conclusions}

An extensive analysis of the accuracy in the theoretical prediction of transmutation and activation cross sections for neutron and proton induced reactions has been carried out. The predictive capabilities of the different models for the description of the nuclear level densities as implemented in the TALYS and in the modified ALICE codes have been investigated by means of the systematic comparison of the theoretical results with experimental EXFOR data relative to neutron and proton induced reactions. Recommendations are provided to the users on the best combinations of codes and models to optimize the accuracy of the simulations.

This work is partially supported by the European Commission through the IP-EUROTRANS project under the contract FI6W-CT2004-516520.

\section{References}

1. D.E. Cullen, A. Trkov, Program X4TOC4, Version 2001-3, IAEA-NDS-80, March 2001.

2. A.J. Koning, S. Hilaire, M.C. Duijvestin, TALYS: Comprehensive nuclear reaction modeling.

3. C.H.M. Broeders, A.Yu. Konobeyev, Yu.A. Korovin, V.P.M. Blann, Forschungszentrum Karlsruhe Report FZKA 7183, 2006; http://bibliothek.fzk.de/zb/berichte/FZKA7183.pdf.

4. A.J. Koning, M.C. Duijvestin, Nucl. Phys. A 744, 15 (2004)

5. A.J. Koning, J.P. Delaroche, Nucl. Phys. A 713, 231 (2003).

6. C.K. Kalbach Walker, PRECO-2000, March 2001; http://www.nndc.bnl.gov/nndcscr/model-codes/preco2000/index.html.

7. M. Blann, H.K. Vonach, Phys. Rev. C 28, 1475 (1983).

8. M. Blann, ALICE-91, Statistical Model Code System with Fission Competition, RSIC CODE PACKAGE PSR-146.

9. C.H.M. Broeders, A.Yu. Konobeyev, Yu.A. Korovin, V.P.M. Blann, Forschngszentrum Karlsruhe Report FZKA 7183, 2006; http://bibliothek.fzk.de/zb/berichte/FZKA7183.pdf.

10. Yu.A. Korovin, A.Yu. Konobeyev, P.E. Pereslavtsev, Progr. Nucl. Energy 40, 673 (2002).

11. A. Iwamoto, K. Harada, Phys. Rev. C 26, 1821 (1982).

12. K. Sato, A. Iwamoto, K. Harada, Phys. Rev. C 28, 1527 (1983).

13. A.Yu. Konobeyev, V.P. Lunev, Yu.N. Shubin, Acta Physica Slovaca 45, 255 (1995).

14. A.V. Ignatyuk, G.N. Smirenkin, A.S. Tishin, Sov. J. Nucl. Phys. 21, 255 (1975).

15. A.V. Ignatyuk, Level Densities, in Handbook for Calculations of Nuclear Reaction Data, Report IAEA-TECDOC-1034 (1998).

16. S. Goriely, http://www-nds.iaea.org/RIPL-2/densities.html.

17. H. Leeb, M.T. Pigni, I. Raskynite, Proc. Int. Conf. on Nuclear Data for Science and Technology, Santa Fe, New Mexico, USA (2004), p. 161.

18. C.H.M. Broeders, A.Yu. Konobeyev, L. Mercatali, Kerntechnik 71, 174 (2006).

19. C.H.M. Broeders, A.Yu. Konobeyev, L. Mercatali, J. Nucl. Rad. Sci. 7, 1 (2006).

20. C.H.M. Broeders, U. Fischer, A. Yu. Konobeyev, L. Mercatali, S.P. Simakov, J. Nucl. Sci. Technol. (2007) (to be published).

21. A.Yu. Konobeyev, C.H.M. Broeders, U. Fischer, L. Mercatali, I. Schmuck, S.P. Simakov, Proton Activation Data File (these proceedings).

22. J.-Ch. Sublet, A.J. Koning, R.A Forrest, J. Kopecky, The JEFF3.0/A Neutron Activation File, EAF-2003 into ENDF-6 format, Report JEFDOC-982 (Nov. 2003). 